accounted for more rationally in this way than in any other.

There are, of course, other general causes tending to produce central asthenopia which might be considered in this connection, but which must of necessity be omitted. It should be noted, however, that while the error of refraction which produces accommodative asthenopia remains nearly the same through life, and while, also, the unequal balance belonging to muscular asthenopia varies comparatively little, on the other hand, those conditions of the general health which accompany ceritral asthenopia do change readily. The application of this fact is practical and familiar. Convex glasses or prisms which once gave the patient relief can be changed for those which are weaker, or are voluntarily laid aside altogether when the health of the patient has improved. In certain cases after we have gone through the usual exact routine with ophthalmoscope, ophthalmometer and various muscular tests, it is true we do succeed in detecting some slight anomalies which have existed perhaps for years, and which are aggravated only temporarily by some such fault in the general condition as has been indicated above, and which the family physician himself has overlooked. Under such circumstances if we then also neglect the general health of the patient and set ourselves at work to correct only the error of refraction or the muscular balance, we may be sure of obtaining little or no improvement at first. But as the patients are patient, and consent to rest, or to exchange a sedentary life for fresh air, or a life of hard work for one with more relaxation, in such cases relief does come, but comes very gradually, and this improvement must be accredited more to nature's tonics and time than to any skill in prescribing glasses or to "exercises," or to any of those measures which in other individuals are undoubtedly of benefit.

I know that this will be considered by many as heresy, but it is none the less the truth. It behooves us to recognize it frankly and to be on our guard accordingly.

Wo have reason to congratulate ourselves upon the advances made during the last few years, especially in America, in the methods of determining and of treat. ing accommodation and muscular asthenopia. But let us beware of rapid progress in these two lines, at the neglect of a third often equally important.

To avoid that, for our own credit, and for the greater comfort of our patients, I venture to recall these facts, already familiar, concerning the relation of the general health to asthenopia.

\section{CHAIRMAN'S ADDRESS.}

Read in the Section on Laryngology and Otology, at the Forty-seventh Annual Meeting of the American Medical Association,

BY G. V. WOOLEN, M.D.

INDIANA POLIS, IND.

Gentlemen of the Section:-It is especially pleasing to me to meet so many of you again, and in this fair city of the South already noted for its activity in promoting the general welfare of mankind.

I again wish to express to you my sincere thanks for the high honor conferred in selecting me to preside over this part of our work so dear to us all. Whatever may be our ideas relative to specialties and the organization of special societies, it must be conceded that the unity of the profession must be preserved, and that much of our best work should be per- formed in connection with the meetings of this Assocration. It is, therefore, fitting that we meet annually with the profession for special as well as general work, and the consideration of matters pertaining to the general welfare of the fraternity.

It will be a sad day for specialists when this is neglected. We can not afford to ignore these claims and I am persuaded that it is the purpose of you who have helped to further the interests of this section from its beginning to have no thought of forgetting this American Medical Association, now old and honored in its usefulness.

In view of the length of our program, and the desire of your Chairman that a full discussion of its merits may be secured, without which our meeting will be robbed of much of its interest and profit, it is not his intention to furnish an extended address. In this respect, and in the preparation of the program it is desired to depart from former customs somewhat, hoping thereby to increase the interest of our meeting.

By collecting papers on a!lied subjects for individual sessions and following them with a speaker specially chosen to open the discussion it is hoped the interest will be direct, and much useful information secured, and thus justify the departure. Indeed it has been a question with me if this department might not be extended by the selection of a single individual to furnish a paper for each session of our future meetings and of one or more to open the discussion and so confine attention, thereby securing more exhaustive work than can be had by our present methods.

Our specialty is young, but its field is broad, vastly more so than the uninitiated can know, and our very best endeavors should be given to bring it up to the high plain of its importance, and not the least of these efforts should be put forth annually in this section here in close contact with our fellows of the other departments.

\section{ORIGINAL ARTICLES.}

\section{SUBCONJUNCTIVAL INJECTIONS.}

Read in the Section on Ophthalmology at the Forty-seventh Annual Meeting of the American Medical Association, held at Atlanta. Ga.. May 5-8, 1896.

\section{BY EDWARD J. BERNSTEIN, M.D.}

BALTIMORE, MD.

Like all other remedial agents, subconjunctival injections must run the gauntlet of ill-considered judgments, both favorable and adverse, until its place shall have been firmly assigned.

Few men, like Abadie, Darier, Deutschmann, Gepner, Schmidt-Rimpler, Pflueger and Zossenheim have yet had sufficient personal experience to entitle them to give conclusive verdicts. In the main, these men assert, we have in this method a means at our hand, swift, sure and intense, which under proper precautions and indications should stand us in good stead, whether employed alone or in conjunction with general treatment.

That it is a panacea or infallible, not even an enthusiast will contend. Much adverse criticism comes from those who declare that we have no precise indication for its employment. Certainly we must, in part, admit this contention, but should one expect a hard and fast demarcation in a method so novel? 
Even Darier, who has been working in this line since 1888, admits that he must still often work in the dark. But are we uniformly successful with opium, quinin, or the bromids? Shall we give up the use of the Eustachian catheter because of discouragement and occasional failure?

Though Rothmund, in 1866, and Segondi, in 1871, were the pioneers in the use of subconjunctival injections, it is to the persistence and zeal of M. A. Darier we owe the present status of the question.

Though rather unfavorably impressed with the method when I observed it in the fall of 1891 under Darier, that opinion has been greatly modified during the past sixteen months. My task shall be to supplement the work of Valude (Annales D'Occulistique, August, 1893) and to record my own experience.

Upon technique I shall only touch, referring for more complete data to the original articles and the many translations, but the following points are worthy of consideration:

1. Observance of every practical aseptic precaution -sterile ground, solution and instruments.

2. Thorough anesthesia of conjunctiva with 4 per cent. (sterile) cocain solution.

3. Use of cyanid of mercury instead of sublimate. Cyanid hydrargyri is compatible with cocain muriate. Adding 1 per cent. of cocain increases anesthetic effect. Cyanid is less irritating than sublimate, and is taken up as such by the tissues instead of being converted into the slowly soluble albuminate,

4. The injection is to be made subconjunctivally, and as far as possible at a tangent with the globe. and not under Tenon's capsule.

We can avoid the larger vessels by simply rubbing the lid over the eye once or twice, when they become visible, and one can readily choose a clear spot. We are not so fortunate with the fine nerve twigs, and should we puncture one of these pain will be quite severe for twenty-four hours.

Muttermilch (Anncles D'Occulistique, September, 1894) asks: "Why resort to injections when we know that fluids reach the interior of the eye by simple instillation into the sac?" Even if sublimate so given were absorbed, which he questions, basing conclusions on Tichomoroff, the dose would be so infinitesimal that its effect would be nil. "Say you inject onetwentieth mg., the beginning dose, two-thirds of the fluid is lost (?) in the conjunctival sac, leaving but one-third, or one-sixtieth mg. (0.000017), to enter the eye. Now reckon the volume of the eye at 7 c.m., you then obtain a dilution of 1 to 400,000 which can hardly be called an antiseptic solution." Furthermore, he believes whatever good results is from suggestion.

To these we may reply that the action of drugs injected is intensified, and it is a more exact method of dosage. Pflueger converted the crystallin lens into an emerald mass by so injecting solution of fluorescin. Cocain thus used produces a rapid and thorough anesthesia of the iris, which simple instillation does very unsatisfactorily, if at all. But it is objected that this does not hold true for sublimate, which is converted into the slowly soluble albuminate in the body. Does this latter not apply equally as well to its hypodermic use, and who can gainsay its superiority in accuracy of dosage, rapidity and intensity of action over the administration per ora? Yet here we use the cyanid, which is not, or but feebly so, transformed. Admit even that all the mercury is so changed, it will none the less be absorbed. Bocchi demonstrated microscopically the presence of mercury in the tissues after injections under the conjunctiva.

The assertion that but one-third of the fluid injected enters the eye is a flight of the imagination and not founded on fact. Let us even admit this to be true, yet are his calculations and conclusions erroneous.

Say we inject hypodermically 0.01 , the usual dose, of $\mathrm{HgCl}_{2}$ for a person of $60 \mathrm{kgm}$. Remember much of the hydrargyrum is carried off by the excretory organs; the liver takes up and retains a larger proportion; then come the other glands, especially the salivary glands; next, the skin, and finally the other organs and tissues. Now, how much of this 0.01 goes to the eye? And have we not all seen specific lesions actually melt away under its influence? Does it not look on the face of it that a larger proportion of hydrargyrum enters the eye by this means than by systemic medication? And is it not also likely that hydrargyrum acts in a much smaller dose than we generally credit? You can not estimate the quantity, as you see, by saying, "If the eye weighs $6 \mathrm{gm}$. and the body $60 \mathrm{kgm}$. then one-ten-thousandth goes to the eye."

I propose during the summer to make the actual experiment, using some such method as suggested by Dr. Rudolph Winternitz, and promise to report later. Are we at all sure that drugs injected subconjunctivally enter the eye? Of this there can be no doubt. "Let us inject a solution of $\mathrm{K}_{2} \mathrm{FeCy}$ subconjunctivally, and after a short time enucleate the eye and fix the eye in an alcoholic solution of ferric chlorid $\left(\mathrm{Fe}_{2} \mathrm{Cl}_{6}\right)$. On making sections we can show that the first solution entered by way of the lymph channels, for these show colorless on a blue background. The connective tissue retains the $\mathrm{K}_{2} \mathrm{FeCy}_{3}$ with great tenacity", (Schwalbe, Anut. Des Auges). Ovis and Pflueger have demonstrated China ink-in the lymph channels -which had been previously injected under the conjunctiva.

As several writers contend that the main action of subconjunctival injection is limited to the anterior portion of the eye, and therefore preferred to inject, if at all, under the capsule of Tenon. Carl Mellinger and Domenico Bossalino determined to find out just how far fluids so injected would enter the eye and its neighborhood; they made a series of injections with sterilized mixtures of China ink. These were well borne and produced no inflammatory reaction. The staining particles were found in the lymph channels, the leucocytes could not be demonstrated as charged with the material to any considerable extent. They showed that the particles did follow the large lymph channels of the whole globe and optic nerve, and that not only was the eye and nerve surrounded by such, but the supra-choroidal spaces (by smaller communicating channels) and the intervaginal spaces (Zwischenscheidenraum) as well.

Sublimate thus injected entered the eye as albuminate. Neither Bach nor Hess could discover any sublimate in the interior of the eyes they experimented on, though Bocchi, Brugnitelli, Gallemaerts and Jolly always recovered small quantities by electrolysis.

Pflueger employs solution trichlorid of iodin ( 1 to $1,000)$. His results in general are not so good as those obtained by the use of hydrargyrum. Still in retinal detachment, macular retino-choroiditis, he reports good results where others meet failure with hydrargyrum. 
Is it not likely an indication to employ iodin trichlorid in some of these cases and hydrargyrum in others? Or are they not at least worthy of trial? Marti following in the line of Mellinger found equally good results from the use of weaker solutions of hydrargyrum. He declares that subconjunctival injections act not through any germicidal influence, but by action on the lymphatic circulation (stimulating its rapidity) thereby causing resorption and elimination of pathologic products, thus hastening healing. That his results with salt were equally as efficacious as with hydrargyrum, with the advantage of greatly diminishing pain, and furthermore avoiding the adhesion of the conjunctiva to sclera at site of puncture

To this Darier replies that adhesions only occur when puncture is made too near the limbus or too deep under the conjunctiva. Pain is a variable quantity even in the same individual. Sometimes in one patient an injection will be perfectly painless, while the next time it will be excessive. He had one patient who went through a whole course of hydrargyrum injection without pain, and who complained bitterly when he once injected distilled water. Darier had tried salt solution, iodin trichlorid, soda salicylate and various other drugs, but is convinced that hydrargyrum is most reliable in his hands.

As soon as Mellinger, Pflueger and the rest prove their assertion by an array of sufficiently conclusive observations, Darier says he will be among the first to admit their utility, and will follow their lead. At any rate, these men obtain good results from this pro cedure, and this is a gain in the right direction. We must not forget that it is not intended to do away with general treatment; all that is claimed for it, is that it intensifies the action of hydrargyrum and hastens cure.

Mellinger declares the action of subconjunctival injections to be alterative; possibly hastening the flow in the lymph channels, thus carrying infectious particles away more quickly. Gepner is satisfied that it is the hydrargyrum which is beneficial, because in two instances he used salt solution with no result; improvement began immediately after substitution of hydrargyrum.

Gutman condemns the method. He says it is not only painful and gives no good results, but often works positive injury. He characterizes Darier's work as insufficient and inaccurate. No one who has observed Darier will question his accuracy or sincerity. As to insufficiency, this is in a measure true, as Darier admits, but consider that under his hands, the work has gone on steadily since 1888, that Deutschmann has made some 2,000 injections, that between Gepner, Peunow, Picunoff, Bergmeister, Schmidt-Rimpler and Grossman some three hundred cases and more are reported, and we must admit that it is not whoily an untried experiment.

Yet, Gutman, from the standpoint of experience gained in twelve cases, would sweep away this whole structure. Let us examine his own report, and we see that his results are better than his conclusion, and that they bear out the utility of this method. He obtained good results with iodin trichlorid; had he continued this remedy doubtless his results would have been better; at any rate we will await the results of his experiments with $\mathrm{NaCl}$. solution and trust they will be more extensive, and that he will come to modify his former opinions.
Gallenga found that corneal ulcerations artificially produced in rabbits' eyes were quickly healed by subconjunctival injection. This, Muttermilch declares is without significance, as such heal quickly when nothing is done, and were it really due to hydrargyrum, he might have obtained equally good results from simple instillation.

According to Muttermilch, "Should one use this treatment for sympathetic ophthalmia, one would make the double mistake of using an innocuous means against a microörganism which does not exist (proceeding from the standpoint of Deutschmann), for he says the microbian theory is not alone not proven, but every pathologic and physiologic experiment, as well clinical experience, is against such a theory. In reference to the two cases myopic choroido-retinitis reported by Gepner as benefited, he is of the opinion that the rest which the patients obtained in the hospital would have done the same. As to prevention of post-operative purulent infection, he has the greatest doubts, for we know even without this new treatment few wounds now suppurate, and that it is rare for iritis to be transformed into irido-choroiditis." In these latter he is in a measure correct, but when purulent infection does start often sad havoc is caused before its progress is checked, and it is right to be on the safe side. We know Jaeger and Arlt had a percentage reaching 95 and 97 in their cataract extractions in pre-aseptic days. Does this release us to-day from taking the most stringent precautions? As a matter of fact, his whole criticism is based on an experience gained in three cases: One ulcus serpens cum hypopyon, one kerato-iritis traumatica and one of ophthalmia sympathetica. In his ulcus serpens case he ruptured the cornea, most likely because he did not observe the rule not to inject too near the limbus, and in the other two he desisted on account of pain. He further attributes many of the reported cures to suggestion. The pain induced and a glance at the list of cases reported cured by this treatment is sufficient to negative any such theory. A criticism based on so wide (sic) an experience can hardly be upheld in the face of so much positive clinical proof to the contrary. My own personal experience was gained from seven cases.

Case 1.-Hypopyon keratitis; J. S., 20 ; recent injury, his anterior chamber one-fifth full of pus; marked photophobia; much ciliary pain; lachrymal ducts normal. Typical case. Patient has been under atropin, pressure bandage and iodo form treatment for sixteen days : is worse and in more pain. Under these circumstances injected one portion of a Pravaz syringe full cyanid of mercury (1-3000); continued the pressure bandage. Next day hypopyon had decreased and he had the first night's sleep since his present illness began. Four days later repeated the injection and four days thereafter the third, when ulcer was covered with epithelium and case went on to recovery under simple bandage.

Case 2.- April 2, 1894, M. L., age 26. Keratitis ulcerativa cum hypopyon. Typical case, in a very much reduced individual. Resulted from having baby stick finger-nail into cornea. Case progressing very unfavorably for eighteen days. Hypopyon beginning; whole cornea cloudy; injection of cyanid on April 20 , followed by three more at intervals of five days. Recovery began from the first injection. Cloudiness clearing up from day to day. Cure with small peripheral macula of cornea.

Case 3.- J. W. Had had several attacks of iritis in each eye; no luetic history : borth irides bound down by many adhesions, the right one almost completely. Could allay pain and inflammation, but could not break up synechia, so performed iridectomy on right eye. Was told to return immediately if any symptoms of trouble in left eye. In six months some exposure brought on another attack of iritis. In the face of his old synechia I feared a total occlusion and offered the alternative of iridectomy or subconjunctival injections. The latter was, 
accepted, and as result of four injections the old adhesions yielded to the atropia and his pupil is now round.

Case 4.-Keratitis punctata. W. T., aged 30. Typical case of acquired luetic keratitis punctata. Had been under care some three weeks and was progressing very slowly. Suggested subconjunctival injections, which were accepted. After five injections at intervals of five or six days, patient could return to work. In this case I can only claim that the injections hastened the progress, as it was beginning to show signs of recovery when I began.

Cases 5 and 6 were cases of optic nerve trouble. One, H. J., positive luetic history. Came when vision of right eye was reduced to light perceptions, of left eye to counting fingers at $6 \mathrm{~m}$. Field of vision narrowed and contracted; color sense also very defective for blues and greens. Typical atrophic dises in both eyes, showing lamina cribrosa. After routine treatment had been instituted for more than six weeks, subconjunctival injections to the number of twelve were administered, but, except for a light transitory improvement, to no avail.

Case 6.-Typical tobacco amblyopia, in which cure was hastened by seven injections and the length of treatment reduced to six weeks.

Case 7.- Is a case of old choroiditis disseminata in left eye, with total loss of vision, and detachment of retina in right. This case is being treated with iodin trichlorid and result will only be known in the future.

Let us now turn to special indications for the employment of subconjunctival medications and see when and by whom they are endorsed.

In keratitis parenchymatosa general treatment is the first and most constant indication. Above all hypodermic injection of sublimate, not neglecting atropin, warm compresses, etc. Special indications calling for subconjunctival injections are keratitis benigna, keratitis circumscripta, keratitis atonica or at least with a very moderate reaction; here the results are absolutely marvelous; with each injection one often sees the gradual recession of the area of infiltration. When limited to the center of the cornea massage with lanolin ointment of mercury produces active resorption of old maculæ. (This I have tried in a number of cases with most excellent results.) At the period of decline, when the bulbar conjunctiva has regained its normal state, these injections will often clear up in a few days what would otherwise have taken months. In the acute, violent pannus of keratitis parenchymatosa all local irritating treatment is absolutely contraindicated, and this is also true wherever there is stasis of the ocular circulation.

Deutschmann and Zossenheim (Beitraege zur Augenh. XV, 1894) agree "that we can often shorten the treatment to four weeks, while it usually takes twice or thrice as long."

Gepner would expect good results, though must acknowledge one of nine cases was cured, the other eight merely benefited.

Peunow treated twenty-three cases with good results, best however in those of specific origin.

Picounoff treated between twenty and thirty; commends treatment under above conditions.

Veasey treated two cases. Cured one, stopped treatment of the other on account of pain.

Abadie endorses all Darier says in this regard.

Chibret, Mellinger and Gosetti have used the method and approved of it.

Motais was "impressed by the rapidity of recovery."

Gerasimos Materangos treated a number of cases of traumatic and infectious keratitis in conjunction with general treatment, best results.

Schmidt-Rimpler treated nine cases, and does not recommend.

Haab, ten cases, no result.

Keratitis ulcerativa cum hypopyon.-Its utility is here questioned because it has not been applied with sufficient discernment.

In mild cases, it will produce cures quicker than by the classic treatment. In those of average intensity the galvano-cautery to the edges of the ulcer is the first indication together with antiseptic dressing. In grave cases where the globe or cornea is threatened Saemisch incision or galvano-cautery, or both are necessary. Cure is hastened when followed by subconjunctival injections (five to ten divisions of the syringe) made as far as possible from the cornea and above all not under Tenon's capsule. In great hyperemia the artificial leech applied to temple helps.

Failure to observe these points produced rupture of cornea in Muttermilch's case. Any such formula as "ulcer of cornea, subconjunctival injections" will result in numerous failures, while observance of the above indications will hasten cure.

Gepner "employs it most frequently in ulcerative forms of keratitis and with best results."

Gragarin-"Good and rapid results up to the clearing of hypopyon; after this not much further improvement is noted."

Peunow-"Considerable help, but does not neglect routine treatment."

Veasey-"As good as other methods."

Abadie-"Highly extols."

Mellinger had brilliant results.

Bocchi - "First injection brought process to a halt." Gossetti- "Tery efficacious from the first injection."

Grossman and Rogman and myself had excellent results.

Chibret - "Resuits doubtful."

Masselon - "Altogether negative."

Schmidt-Rimpler-In thirteen cases of ulcerative keratitis hypopyon. Good results in three. Questionable in eight. Two of simple ulcerative keratitis gave negative results. He thinks very little of it in this latter and hypopyon keratitis.

Deutschmann prefers galvano-cautery, which in his hands works surer and quicker.

In keratitis lymphatica, Darier, Doufer and Segondi coincide that the yellow ointment fills every indication except in grave cases, when the new method gives good results.

Coppez and Gallemaerts give preference in vascular keratitis to subconjunctival injection of potassium iodid solution.

In iritis the indications and the contraindications are very subtle. While in many the results are beneficial, in others it is not only useless, but positively harmful. Whenever iritis is lighted up with violence, and is accompanied by brisk reaction (deep pericorneal hyperemia, chemosis, photophobia, etc.) an energetic antiphlogistic treatment is the first indication in comnection with general treatment of the proper character. Only after the violence has abated is subconjunctival injection indicated. Failure to observe this might provoke an aggravation of symptoms in an eye already inflamed, and is no doubt, the cause of much of the reproach heaped upon this method and the consequent ill success, whereas those who have instituted treatment in relatively benign cases, either at their beginning, their relapse or their decline have according to Darier obtained conclusive evidence of its efficacy, but in benign cases, the older methods are less annoying and often equally as satisfactory.

I coincide with Gepner, who finds its main indica- 
tion in assisting to break up old iritic synechiæ, or in the very beginning of the malady, for as Zossenheim says it cures so quickly that complications have no chance to manifest themselves. It may be used with advantage according to both these men in occlusion of the pupil and where we find hypopyon in the anterior chamber. Peunow treated twenty-eight cases with good results. Picounoff also had the same uniformly good results in a large number of cases. Veasey seven cases, prompt and effectual results in all, as also in cases of chorio-retinitis.

Schmidt-Rimpler nine cases. Cured two cases of plastic iritis, other results divided as follows: One cured after thirteen injections; four relatively good results; two slightly benefited; he recommends further use of this method in iritis and irido-choroiditis.

Grossman, Alt and Mellinger also commend its use.

Bergmeister two cases. Both cured after the fourth injection.

Bocchi and Masselon do not recommend it in iritis.

Irido-choroiditis.- Whatapplies to iritis applies with greater force in this. In a disease so difficult to treat and holding out such poor promise, we often proceed as though groping in the dark, for at times we see one of those eyes apparently quiescent, react with an extreme violence to the slightest local irritation. These are the cases in which we are often led to prematurely perform iridectomy.

With injections one is often surprised to observe not only amelioration, but at times cure, where one would not expect such. In certain grave cases, we are obliged to keep the patient under continuous treatment, often alternating between general and local, so as to give the patient a relative rest.

Grossman treated two cases with good results. Alt also reported success. Pflueger treated his cases with iodin trichlorid and reports: one case of serous iritis, result beneficial; in another no effect; in a third stopped on account of pain and increasing intra-ocular tension.

Matarangas--Good results in conjunction with generai treatment.

Deutschmann-Iritis specifica, as well as non-specifica, this method was at its best. He uses no atropin. Posterior synechia disappeared, and in four to eight days the pupil was quite round, dilating and contracting freely and the eye quiet. Saw no recidives; completed the treatment with mercury internally.

Choroiditis and retinitis.--When structural lesions have occurred, it is impossible to speak of cure in the sense of complete restoration. Much can be done for those who seek our aid early, to avoid such destruction by active and prompt methods, among the foremost of which stand subconjunctival injections.

Often an appreciable effect is noted from the first or second injection, and again not until a great num. ber have been made. In macular choroiditis before central vision has been irremediably destroyed, we can restore if not normal vision, at least bring a considerable amelioration, and in so short a time there can be no doubt of the relation of cause and effect. Darier claims favorable result in at least 25 per cent. of his cases, and in 10 per cent. a result which "I can call surprising.'

Abadie claims its efficacy in all degrees of chronic chorio-retinitis. He condemns the use of potassium iodid alone or with mercury as manifestly injurious. Injections of pilocarpin had no effect except when due to myopia ; certainly not in infectious forms. In obsti- nate cases he uses subcutaneous injections in addition.

Venneman-Results excellent.

Grandclermont-Best effect in serious or desperate affections of middle (vascular) coats of the eye. Above all in irido-choroiditis.

Gepner--Improvement in many cases of chronic choroiditis. Decided in two cases of myopic choroiditis. Peunow-Thirty-one cases choroiditis cured by these means.

Deutschmann--Especially good results in specific chorio-retinitis. In non-specific choroiditis best results from potassium iodid kept up for months.

Schmidt-Rimpler-One good result in choroiditis; two doubtful in chorio-retinitis; thinks this is one of the conditions in which it is worthy of further trial. Bergmeister-Not specially praiseworthy but recommends further trial.

Alt-Good results in choroiditis exudativa and centralis.

Bocchi-No specially good results; in this he is sustained by Masselon, Lacquer, Dianoux.

Bull-Positive effect in allaying severity of symptoms, and shortening the duration of acute iridochoroiditis non-specifica.

Seggel reports a cure in irido-cyclitis.

-Optic Nerve.-Darier says in all infectious inflammations of the nerve, results are encouraging often after classic treatment proves unavailing. In retro-bulbar neuritis often excellent results, though in hereditary form we secure neither better nor worse results than by other means. Does not include tobacco and alcoholic amblyopia which tend to recovery sublata causa. Where actual atrophy of nerve fibers has set in it is useless to expect results. In retro-bulbar neuritis of rheumatic (?) origin, better results than by salicylates or mercury.

In compression neurites, obtained very rapid cure. In specific neuritis, and in one secondary to chorioretinitis greater amelioration than by old method.

We may expect good results in recent infectious inflammations.

"No results to be expected in gray atrophy of tabes, in white atrophy following old inflammatory processes occasionally a slow increase of vision is manifest, but generally only transient."

Grossman, De Wecker, Lindsay Johnson and Matarangas corroborate these opinions. Deutschmann's results negative in every case. In commenting on this in his journal, Hirschberg reports a good result in chronic cptic neuritis, also in one of recent origin.

In sympathetic ophthalmia, Darier found subconjunctival injections available in a number of cases characterized by uveitis.

Abadie says: If the injury is not so serious as to preclude all possibility of recovery, the surface of the wound should be touched with the galvano-cautery, and hydrargyrum bichlorid injected under the conjunctiva. It is often thus possible to arrest sympathetic ophthalmia already declared in the other eye. but if the traumatism is such that there is no hope of saving, or if disorder continues in spite of all these means, we should enucleate the offending eye. The other one will be benefited by the injections. Personally, I should hesitate to temporize with an injured eye which is likely to cause sympathetic ophthalmia. We all know how many such eyes may remain quiescent for as long as fifteen or twenty years, to suddenly light up inflammation in the other eye. 
Gosetti reports remarkable success in a severe and recurrent case.

Deutschmann also reports good results in a number of cases.

Picounoff especially recommends it, here, where we can usually avoid enucleation.

In scleritis De Schweinitz, Bull, Veasey and others report some good results among a number of negative ones.

Many, Abadie and Coppez among the number, declare that they have thus avoided panophthalmitis in many cases of cataract and iridectomy operations, and indeed have checked the disease when it had actually begun. Rogman corroborates this latter statement.

Gepner says he resorts to subconjunctival injections in all cases of serious injury where there is still hope of saving the globe, for two reasons: 1 , because we can not foretell where we have wound infection before we see our patient; 2 , because in every deep injury we can not foretell whether sympathetic ophthalmia will result. Germicidal agents in the lymph channels lessen that danger. Seggel reports cure of suppuration of vitreous after cataract extraction, one case of threatened destruction of the whole cornea and vitreous from infected wound with prolapsed iris, and also cure of a case of orbital phlegmon.

Deutschmann says: "As a preventive of postoperative infection it is of a special utility. Cases that usually resulted in loss of the eye were by this means saved useful vision." In this he is upheld by Gepner and others. He further says: "We can not compare results of experiments made on animals to clinical results in man. In animals large deposits are at once introduced into the eye, which condition bears no relation to that in post-operative infection."

\section{prscussion.}

Dr. G. E. De Schweinitz, Philadelphia--I have used the subconjunctival injections since 1892 and also advised my chief of clinic, Dr. C. A. Veasey, whose results have been quoted, to employ them. My experience is that exactly the same results are obtained whether the bichlorid of mercury or the physiologic salt solution is used, each being equally efficient in suitable cases. I have secured good results in iritis, no matter what its type, provided there is no high inflammatory action. Good results were also obtained in episcleritis and some types of keratitis. I have failed, however, to secure good results in corneal ulcers and in diseases of the deeper coats, e.g., choroiditis. I would call attention to the promptness with which these injections, either salt or mercuric, relieve pain and advance resolution in certain cases of inflammatory disease of the iris and episcleritis, but would also suggest that a relapse is more likely to occur than when ordinary constitutional measures are thoroughly employed. I believe that subconjunctival injections deserve a permanent place in ophthalmic therapeutics, but not to the exclusion of constitutional measures.

Case 1.-A. M., a male Italian, about 40 years of age, pre sented himself at the eye dispensary of the Jefferson hospital for treatment of sore eyes. Upon examination he was found to have a double syphilitic iritis, plastic in character, the initial lesion having been present four months. There were present the usual symptoms of marked pericorneal injection, intense pain, photophobia, lachrymation and a contracted pupil with posterior synechiæ, these symptoms having been present, accord ing to the patient's statement, for four days. In the right eye the iris is attached to the lens capsule by its entire pupillary border, except a small portion in the upper and outer quadrant, this being the only part that would dilate with atropin. The media were hazy, and there was an indistinct view of the fundus which showed an oval disc, with rather large veins, flled with dark blood, but there were no gross lesions. In the

left eye there was also almost complete annular attachment of the iris, there being a small free portion on the temporal side, while the ocular fundus presented practically the same appearance as the other eye. His vision was $10-200$ in each eye. A tropin was instilled for twenty-four hours without any improvement in the pain, and with very limited dilatation in the pupils. He was then given a subconjunctival injection of mercuric chlorid $(5$ minims of a 1 to 2,000 solution $)$ in one eye, and a subconjunctival injection of sodium chlorid (2 to 10 of a 1 per cent. solution) in the other eye, and returned on the following day with the pupils dilated ad maximum, the pain entirely gone, and said that he had spent the night free from pain, the first for about a week. There was absolutely no difference to be detected between the effect on the two eyes. This treatment was continued, he being given an injection of mercuric chlorid in one eye and sodium chlorid in the other, at intervals of two or three days, until he had received five injections of each, no other medication being employed except the solution of atropin. At the expiration of this time the pupils were completely dilated, there had been no pain since the first injection, and it was impossible to say that there had been the slightest difference in the results of the two solutions. His vision was 20.30 in each eye, there being some pigment on the capsule of the lens where the iris had been attached.

Case 2.-C. B., a male, aged 35 years, came to the eye dispensary of the Jefferson Medical College Hospital complaining of sore eyes that had been present for two days. Upon examination he was found to have a syphilitic plastic iritis in the right eye, the initial lesion having been present about six months. There was a small synechia up and out, the vision was $20-100$ and there were the usual symptoms of the disease present. In the other eye there was a slight conjunctivitis, the vision being 20-20. He was suffering from severe pain and was given an injection beneath the conjunctiva of the solution of sodium chlorid; atropin was instilled into the eye, and he returned on the following day entirely free from pain, the pupil dilated, the peri-corneal injection much less and the photopho bia less severe. He was given similar injections on alternate days until he had had four, no other treatment being employed beyond the instillation of the atropin solution, when the inflammatory condition of the eye had entirely disappeared. No dif ference could be observed between the promptness with which the disease yielded to the injections of sodium chlorid and the promptness with which other cases had yielded to the injections of mercuric chlorid. The vision in this case returned to the normal.

Case 3.-H. F., male, aged 31 years, consulted me in February of this year for a rheumatic iritis. There was no specific history, and he had had several attacks of the same character before, each time being treated by a competent ophthalmic surgeon who had pronounced it rheumatic in character and relieving it each time by the use of the salicylates, in addition to other treatment. The pain was intense, and desiring to see what effect an injection of salt solution would have, one was made at once, and other treatment, excepting the instillation of atropin, withheld. On the next day he returned with a complete cessation of pain, except when exposed to bright light, the pupil entirely dilated and a marked reduction in the inflammatory condition. He was given five other injections, after which the iritis was practically well, when he was placed on the anti-rheumatic treatment. Other cases of iritis, syphilitic or rheumatic, treated by myself with injections beneath the conjunctiva of mercuric chlorid, have not shown any advantage over the injections of the solution of sodium chlorid. Indeed, it is my experience that the latter causes less pain, and relieves the pain of the iritis just as speedily, if not more so, than the injections of the mercuric chlorid.

Dr. G. C. SAvagE, Nashville--I have used only the bichlorid, but found it too painful. When opportunity offers I shall use the cyanid of mercury. I wish to condemn the practice of not using atropin in iritis, although subconjunctival injections of cyanid of mercury may be capable of accomplishing much. Atropin should never be omitted in the treatment of iritis, whatever else may be done. I want to go on record as believing that it would be a grave error to neglect bringing the iris under the influence of atropin, whoever may recommend to the contrary.

DR. D. S. Reyrolds, Louisville-I am not persuaded that any local method of treating iritis, except the persistent use of an efficient mydriatic, has ever accomplished any desirable result. The subconjunctival injection of a solution of chlorid of sodium in cases of synechia following iritis from all causes 
is undoubtedly valuable, but I should certainly avoid the use of any subconjunctival injections during the active stages of any form of iritis.

Dr. A. W. Stirling, Atlanta-I watched the use of this method in a fair number of cases in London and Paris and have tried it myself, but gave it up because it was so painful. It will not take precedence over the older methods.

DR. A. R. BAKER, Cleveland-Having used the strong solutions of bichlorid of mercury and found the injections painful, I estimated the amount of mercury which would reach the eye from a subcutaneous injection as generally used, and determined that a much weaker solution would probably prove efficient, I tried a 1 to 10,000 solution with excellent results in a large number of cases, notably interstitial keratitis and central choroiditis. The injections were never painful and the results appeared as good as from the stronger solution. In private practice it has been found necessary to combine its use with the accepted methods of treatment, but with more rapid recov ery than when its use is omitted.

Dr. Dunbar Roy, Atlanta-I use the method largely in my clinic, where the patients consist largely of the colored race, who have a great deal of corneal trouble. In ulcers of the cornea I have had good results, but no better than when I use hot fomentations and iodoform. In post-suppurative trouble and panophthalmitis and in cases of suppurative iritis $I$ have used it with success. I have used 1 to 1,000 bichlorid with an ordinary hypodermic needle, sterilizing both the instrumen and the field before the operation. In iritis $I$ have not seen adhesions break up and have had no results in choroiditis, but my experience leads me to say that in ulcerative and suppurative forms of conjunctivitis or keratitis the results are as good as those obtained by other methods.

Dr. E. J. Bernstern - I have no experience as yet with relapses, but one must not forget that neither atropin nor gen eral treatment are to be neglected in iritis. As to pain, my patients had none, or very little, as the result of subconjunctival injections. I am now treating a case of detachment of the retina by this means without much hope of cure, but because no other treatment has availed. I believe no one should follow A badie in not using atropin in iritis. I should hesitate too implicitly to follow his lead. In phlyctenular keratitis I should not think of using this method, as I believe most men agree that the yellow ointment is all that is needed.

\section{EXTRA-DURAL ABSCESS FROM MASTOID EMPYEMIA.}

Read in the Section on Laryngology and Otology at the Forty-seventh Annual Meeting of the American Medical Association

held at Atlanta, Ga.. May 5-8, 1896.

BY B. ALEXANDER RANDALL, M.D.

PHILADELPHLA, PA.

When the presence of a "mastoid abscess," declared by the occurrence of a fluctuating collection of pus on the surface of the skull behind the ear, only caused the surgeon to incise and evacuate the abscess, we heard little of extra-dural or cerebral abscess except as a curiosity of the postmortem room. Even when the farther step of opening the mastoid became more common, it was so often merely in the interest of better evacuation and drainage that exploration for the remoter extensions of the lesions was rare. It is only since we have more generally adopted the idea urged by all experienced operators, that every possible trace of the pathologic condition shall be extirpated at the operation in the effort to secure immediate cure, that the relative frequency of extra-dural abscess has been recognized. The earlier operators looked upon the middle cerebral fossa and the sigmoid sulcus as regions dangerous to approach, and perhaps held their hands as they followed some purulent track lest it should lead them into these dreaded cavities. The impetuous entered them oftener than they meant, and the careless oftener than they knew, while the careful and conscientious surgeon was gradually learning the innocuous and beneficent effect of hunting down the disease even into these and deeper regions. With the abandonment of the trephine and drill in favor of the gouge and spoon, much of this fear has died a natural death. There is less chance of unintentionally and blindly penetrating the inner table of the mastoid and wounding or infecting the important structures within. Mastoid anatomy is being better learned and the rule has grown more common to operate in each case as though the relations were the most disadvantageous possible, and to assume no safety that has not been demonstrated. And with this knowledge that the middle lobe of the brain may extend down as low as the upper margin of the meatus and the lateral sinus be separated by hardly a film of bone from the back wall of the canal or the mastoid surface at the usual point of attack, there has grown a proper confidence in the wisdom of opening the intracranial cavity in appropriate cases. It should always count as a bungling step when accidentally these cavities are opened, or an officious one when needless; but the futility of half measures grows more evident with experience. One who formerly looked askance at the radical procedures and views of Schwartze and others who chisel open the mrstoid in scores of cases yearly, finds his tendency to follow this lead to be limited principally by his lack of a like material. And since the grippe epidemics few of large aural practice but have had growing opportunities to learn the need of such measures.

We can no longer look upon "mastoid trephining" (as we may still call the operation whether done with chisel, burr or spoon) as permissible only as a lifesaving step; nor can we rest content to leave to long after-treatment the completion of the cure which might have been more safely as well as more quickly secured by more heroic thoroughness. It is to be hoped that American aural surgeons will continue truly conservative in their choice of cases, methods and efforts after thoroughness, eliminating all cases that might be cured by less radical procedures and sacrificing to brilliancy of immediate result no function that patience might have saved; but it is also to be hoped that they will give little basis to the charge sometimes made by the surgeon, that they are too timid in their work and shrink from doing their full duty when half measures are insufficient.

The past decade has seen the publication of hundreds of cases in which there has been extension of caries from the tympanic inflammation to the dural surface of the temporal bone, with pachy-menigitis and outpouring of pus between the bone and dura. In itself it constitutes no great menace to life, and the prognosis after drainage is generally excellent. So little may be the head symptoms caused by it that its presence is a total surprise to the operator; and one marvels that lesions so extensive may be wholly undeclared by the usual signs.

Surprisingly innocuous as the extra-dural abscess has generally proved if drained outward by nature or art, the story is wholly different in no small group of cases. Most of the thrombotic lesions of lateral, petrosal or cavernous sinus have been secondary to extra-dural abscess. Many cerebral and cerebellar 